\title{
Jacobi elliptic function solutions to variable nonlinear Klein-Gordon equation
}

\author{
Chunhuan Xiang ${ }^{1}$, Bo Liang ${ }^{2}$, Longcong Chen ${ }^{2}$ and Honglei Wang ${ }^{2, *}$ \\ ${ }^{1}$ School of Public Health and Management, Chongqing Medical University, Chongqing \\ 400016, P.R. China \\ ${ }^{2}$ Faculty of basic medical science, Chongqing Medical University, Chongqing 400016, P.R. \\ China \\ *Corresponding author, Email:w8259300@163.com
}

Keywords: Jacobi elliptic function, variable nonlinear Klein-Gordon equation, travelling wave solutions, numerical simulations.

\begin{abstract}
By means of the extended mapping method, the traveling wave solution for the variable nonlinear Klein-Gordon equation is investigated, which is obtained in terms of the Jacobi elliptic functions. The hyperbolic function solutions and trigonal solutions are also obtained. The numerical simulations are attached. At the same time, the physical meanings of the obtained solutions are discussed, and the problem needed to further study is pointed out.
\end{abstract}

\section{Introduction}

The characteristics of nonlinear phenomena in various physics fields can be mathematically described by nonlinear evolution equations. Among them of great physical significance are the equations that possess soliton solutions. The nonlinear Klein-Gordon equation generalizes the linear relativistic equation for a charged particle in an electromagnetic field derived by Klein [1] and Gordon [2]. In nuclear and high energy physics the study of exact solution of the Klein-Gordon equation (KGE) is of high importance for mixed scalar and vector potentials, which is also as a model for a self-interacting, nonlinear scalar field in quantum field physics. The stability of periodic traveling wave solutions to the nonlinear KGE has been investigated by many researchers using various techniques and under different perspectives[3-8].

Generally, the extended mapping nonlinear KGE is read as

$$
u_{t t}-\kappa u_{x x}+V^{\prime}(u)=0 \text {, }
$$

where the subscripts denote the partial derivates of $x$ and $t$, the potential $V$ is a real function. The potentials are important in the theory of the Klein-Gordon equation, which are applied in magnetic fluid theory[9], nonlinear meson theory[10], solid state physics[11], and in classical sine-Gordon and $\phi^{4}$-field theories[12]. For polynomial potentials $V$, Chicone's theory is particularly convenient to apply to determine whether a family of librational orbits that surround a stable fixed point. One may consider cubic nonlinearities arising from a quartic double-well potential:

$$
V^{\prime}(u)=\alpha u-\beta u^{3} \text {. }
$$

Then, the variable nonlinear Klein-Gordon equation is rewritten

$$
u_{t t}-\kappa u_{x x}+\alpha u-\beta u^{3}=0 ;
$$

where $\kappa, \alpha$ and $\beta$ are nonzero constant parameter, $u$ represents a real scalar function $u(x, t)$. The remaining structure of this Letter is organized as follows: Section 2 is a brief introduction to the Jacobi elliptic function and its Properties. In Section 3, by implementing the Jacobi elliptic function method, some new traveling wave solutions for nonlinear KGE are reported. A conclusion and future directions for research are all summarized in the last section. 


\section{The Jacobi elliptic function and its Properties} form

Consider a general nonlinear partial differential equation with independent variables $x$ and $t$ in the

$$
Q\left(u, u_{x}, u_{t}, u_{x t}, u_{x x}, u_{t t}, \ldots\right)=0 \text {. }
$$

By using the wave variable $\varepsilon=x+w t$ carries eq.(3) into the following ordinary differential equation

$$
Q\left(u, u_{\xi}, u_{\xi \xi}, u_{\xi \xi \xi}, \ldots\right)=0
$$

where $u_{\xi}, u_{\xi \xi}, \ldots$ denotes the derivative with respect to the same variable $\varepsilon$.

Generally, the scalar function $\mathrm{u}$ in terms of perturbation method can be expressed as

$$
u=K_{0}+\sum_{i=1}^{n} p^{i-1}\left(K_{i} p+S_{i} q\right),
$$

where $p$ and $q$ are Jacobi elliptic functions about $x$ and $t ; K_{i}, S_{i}$ are constant parameters; $n$ is fixed by balancing the linear term of the highest order derivative with nonlinear term. $p$ and $q$ satisfy the following equation

$$
\begin{aligned}
& p_{\xi \xi}=f p+g p^{3} ; \\
& q_{\xi \xi}=q\left(a_{1}+a_{2} p^{2}\right) ; \\
& q^{2}=a_{3}+a_{4} p^{2},
\end{aligned}
$$

where $f, g$ and $a_{i}(i=1,2,3,4)$ are to be determined parameters.

The Jacobi elliptic functions have the properties[13-17]:

$$
\begin{aligned}
& \operatorname{sn}(\xi)^{2}=1-c n(\xi)^{2}, m^{2} \operatorname{sn}(\xi)^{2}=1-d n(\xi)^{2}, \\
& \operatorname{sn}(\xi)_{\xi}=c n(\xi) \operatorname{dn}(\xi), \operatorname{cn}(\xi)_{\xi}=-\operatorname{sn}(\xi) d n(\xi), \\
& d n(\xi)_{\xi}=-m^{2} \operatorname{sn}(\xi) \operatorname{cn}(\xi),
\end{aligned}
$$

where $m(0<m<1)$ is the modulus of the elliptic function. The modulus substantially affects the Jacobi elliptic solutions, which will asymptotically go into hyperbolic functions and trigonometric functions when the modulus $\mathrm{m} \rightarrow 1$ and $\mathrm{m} \rightarrow 0$, respectively.

$$
\begin{gathered}
m \rightarrow 1, \operatorname{sn}(\xi) \rightarrow \tanh (\xi), \operatorname{cn}(\xi) \rightarrow \operatorname{sech}(\xi), \operatorname{dn}(\xi) \rightarrow \operatorname{sech}(\xi) \\
m \rightarrow 0, \operatorname{sn}(\xi) \rightarrow \sin (\xi), \operatorname{cn}(\xi) \rightarrow \cos (\xi), \operatorname{dn}(\xi) \rightarrow 1
\end{gathered}
$$

\section{Jacobi elliptic function solutions to nonlinear Klein-Gordon equation}

Consider the nonlinear Klein-Gordon equation in the form of eq.(3) with $\varepsilon=x+w t$, we obtain

$$
\left(w^{2}-\kappa\right) u_{\xi \xi}+\alpha u-\beta u^{3}=0 .
$$

By selecting $n$ to balance the derivative term of the highest order and nonlinear term, we have $n=1$ obtained from eq.(12). Then, Eq. (6) reduced as

$$
u=K_{0}+K_{1} p+S_{1} q
$$

Substituting eq.(13),(7)-(9) into the equation (12), collecting all terms with the same power of $p q$ together and equating each coeffcient to zero, yields a set of simultaneous algebraic equations as follows: 


$$
\begin{aligned}
& \alpha K_{0}-K_{0}^{3} \beta-3 K_{0} S_{1}^{2} \beta c_{3}=0 ; \\
& \alpha S_{1}-3 K_{0}^{2} S_{1} \beta-S_{1}^{3} \beta c_{3}-S_{1} c_{1} \kappa+S_{1} c_{1} w^{2}=0 ; \\
& \alpha K_{1}-3 K_{0}^{2} K_{1} \beta-3 K_{1} S_{1}^{2} \beta c_{3}-K_{1} f \kappa+K_{1} f w^{2}=0 ; \\
& -3 K_{0} K_{1}^{2} \beta-3 K_{0} S_{1}^{2} \beta c_{4}=0 ; \\
& -3 \beta K_{1}^{2} S_{1}-c_{4} S_{1}^{3} \beta-S_{1} c_{2} \kappa+S_{1} c_{2} w^{2}=0 ; \\
& -\beta K_{1}^{3}-3 c_{4} S_{1}^{2} K_{1} \beta-K_{1} g \kappa+K_{1} g w^{2}=0 ; \\
& \beta K_{0} K_{1} S_{1}=0 .
\end{aligned}
$$

Solving the algebraic equations, we get the results:

Case $1 \quad K_{0}=0$ and $4 c_{1} c_{4}+3 g c_{3}=4 f c_{4}+c_{2} c_{3}$, we have

$$
K_{1}= \pm \sqrt{\frac{\left(c_{1} c_{4}-c_{2} c_{3}\right)\left(\kappa-w^{2}\right)-c_{4} \alpha}{3 c_{3} \beta}} ; S_{1}= \pm \sqrt{\frac{\alpha-c_{1} \kappa-w^{2}+c_{1} w^{2}}{3 c_{3} \beta}} .
$$

Using eq.(13), $p=c n(\varepsilon ; m)$ and $q=d n(\varepsilon ; m)$, the traveling wave solution for eq.(3) is given as

$$
u=K_{1} c n(\varepsilon, m)+S_{1} d n(\varepsilon, m)
$$

where $\varepsilon=x+w t$, the numerical simulation for plus sign with $m=0.1$ and $t=-50,0,50$ are shown in Fig.1. The numerical simulation for plus sign of eq. (15) with $p=1 / d n(\varepsilon ; m), q=\operatorname{sn}(\varepsilon ; m) / d n(\varepsilon ; m)$, $m=0: 1$ and $t=-50,0,50$ are shown in Fig.2.

When $p=s n(\varepsilon ; m), q=c n(\varepsilon ; m), m=0.0000000001$, the solution eq.(15) degenerates to the trigonometry functions:

$$
u=K_{1} \sin \varepsilon+S_{1} \cos \varepsilon
$$

The numerical simulation for plus sign of eq.(16) with $t=-50,0,50$ are shown in Fig.3.

When $p=s n(\varepsilon ; m), q=c n(\varepsilon ; m), m=0.9999999999$, the solution eq.(15) degenerates to the hyperbolic function:

$$
u=K_{1} \tanh \varepsilon+S_{1} \sec h \varepsilon
$$

The numerical simulation for plus sign of eq.(17) with $t=-50,0,50$ are shown in Fig.4.

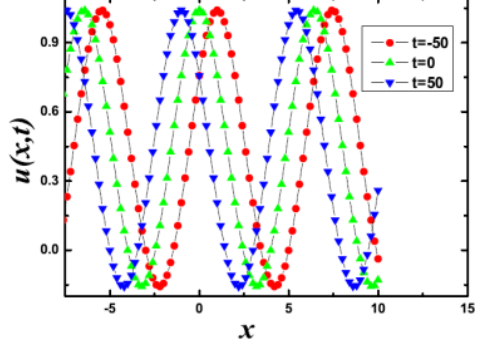

Fig. $1 \mathrm{~m}=0.1$

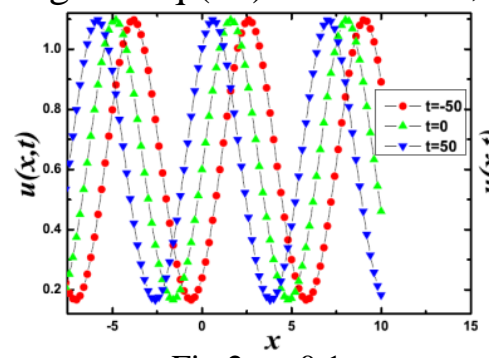

Fig. 2 m=0.1

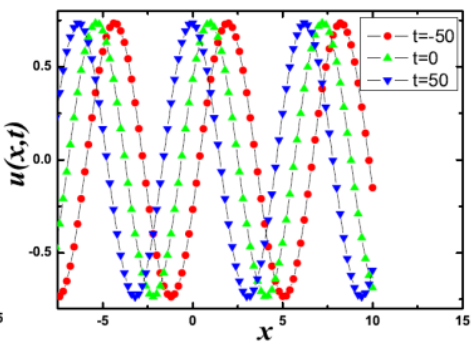

Fig. $3 \mathrm{~m}=0.0000000001$

Case $2 \quad c_{4} \alpha=\left(c_{1} c_{4}-c_{3} c_{2}\right)\left(\kappa-w^{2}\right)$ and $K_{0}=0, K_{1}=0$ we have

$$
S_{1}= \pm \sqrt{\frac{c_{2}\left(w^{2}-\kappa\right)}{c_{4} \beta}} .
$$

Using eq.(13), $q=c n(\varepsilon, m)$, the traveling wave solution for eq.(3) is given as

$$
u= \pm \sqrt{\frac{c_{2}\left(w^{2}-\kappa\right)}{c_{4} \beta}} c n(\xi, m),
$$

where $\varepsilon=x+w t$, the numerical simulation for plus sign of eq.(15) with $m=0.1$ and $t=-50,0,50$ are shown in Fig.5. The numerical simulation for plus sign of eq.(15) with $q=d n(\varepsilon, m), m=0.1$ and $t$ $=-50,0,50$ are shown in Fig. 6 .

All the numerical simulations are given with $c_{1}=-3 m^{2}, c_{2}=2 m^{2}+1, c_{3}=-4 m^{2}-4, c_{4}=m^{2}+1, \alpha=$ $3, \beta=-4, \cdot \kappa=5, w=0.02, f=2 m^{2}-1, g=-2 m^{2}, r=1-m^{2}, x \in[-7.5,10]$, all the figures are obtained in the plot form with the aid of Origin software. 


\section{Conclusions}

The variable nonlinear Klein-Gordon equation in the form eq.(3) have been further studied by means of the extended mapping Jacobi elliptic function method. A variety of periodic waves solutions (15)-(19) of eq.(3) are obtained in terms of Jacobi elliptic functions, trigonometric solutions and hyperbolic solutions, which can be employed to discuss some interest physical phenomena. The performance of Jacobi elliptic functions method is reliable and effective, which gives more solutions. A modified Klein-Gordon equation appears for instance in the study of long Josephson junctions between superconductors when dissipative effects are taken into account.

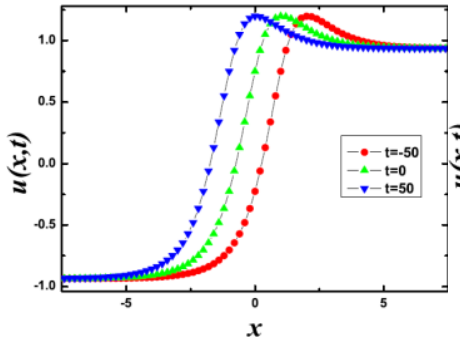

Fig. $m=0.999999999$

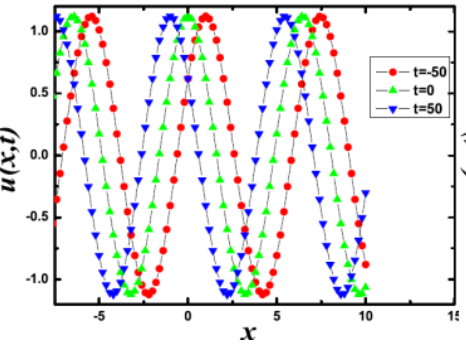

Fig.5 $m=0.1$

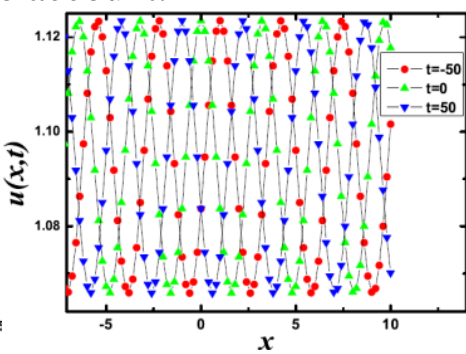

Fig. $6 m=0.1$

\section{Acknowledgements}

This work was financially supported by the Science Foundation of Chongqing City Board of Education (KJ121206)

\section{References}

[1] O. Klein, Elektrodynamik und Wellenmechanik vom Standpunkt desKorrespondenzprinzips, Z. Phys. 41 (1927), 407-442.

[2] W. Gordon, Der Comptoneffekt nach der SchÄodingerschen theorie, Z. Phys. 40 (1926), 117-133.

[3] C. K. R. T. Jones, R. Marangell, P. D. Miller, and R. G. Plaza, On the stability analysis of periodic sine-Gordon traveling waves, Phys. D 251 (2013), 63-74.

[4] M. Badiale, S. Rolando, A note on vortices with prescribed charge, Adv. Nonlinear Stud., 12 (2012), 703-716

[5] Y. Wang, B. Wang, High-order multi-symplectic schemes for the nonlinear Klein-Gordon equation, Appl. Math. Comput., 166 (2005), 608-632.

[6] L. Vu-Quoc, S. Li, Invariant-conserving finite difference algorithms for the nonlinear Klein-Gordon equation, Comp. Meth. Appl. Mech. Eng., 107 (1993), 341-391.

[7] C. Bonanno, Existence and multiplicity of stable bound states for the nonlinear Klein-Gordon equation, Nonlinear Anal., 72 (2010), 2031-2046.

[8] V. Benci, N. Visciglia, Solitary waves with non vanishing angular momentum, Adv. Nonlinear Stud., 3 (2003), 151-160.

[9] S. K. Malik and M. Singh, Modulational instability in magnetic uids, Quart. Appl. Math., 43 (1985), 57-64.

[10] L. I. Schiff, Nonlinear meson theory of nuclear forces I. Neutral scalar mesons with pointcontact repulsion, Phys. Rev., 84 (1951), 1-9.

[11] A. R. Bishop, J. A. Krumhansl, S. E. Trullinger, Solitons in condensed matter: a paradigm, Phys. D, 1 (1980), 1-44.

[12] V. G. Makhanokov, Dynamics of classical solitons (in nonintegrable systems), Phys. Rep., 35 (1978), 1-128.

[13] K. Chamdrasekharan, Elliptic Functions Springer-Verlag, Berlin, 1985. 
[14] V. Prasolov and Y. Solovyev, Elliptic Functions and Elliptic Integrals, American Mathematical Society, Providence, 1997.

[15] H. Wang, C. Xiang, Jacobi elliptic function solutions for the modified Korteweg-de Vries equation, Journal of King Saud University-Science, 25 (2013), 271-274.

[16] G.-T. Liu, Lame equation and asymptotic higher-order periodic solutions to nonlinear evolution equations, Appl. Math. Comput., 212 (2009), 312-317.

[17] L. Liu, Exact travelling wave solutions for the Nizhnik-Novikov-Veselov equation, Appl. Math. Comput., doi.org/10.1016/j.amc.2010.12.033.7 